Results Of the 169 patients identified, 96 (56.8\%) had died, 22 $(13.1 \%)$ had been transplanted while the remaining 51 were alive. The median survival time was 18.8 months. The factors predictive of death were a higher serum AST $(p<0.04)$, ALP $(p<0.02)$, and sodium $(p<0.02)$ and an increased INR $(p<0.01)$. Survival rates were higher in patients of non-British white ethnic origin $(p<0.00)$. Of the 27 patients available for review, 21 (78\%) had some degree of $\mathrm{HE}$ although less than $30 \%$ were on anti-encephalopathy treatment. The factors predictive of HE were older age $(p<0.01)$, with the risk increasing by $6.5 \%$ for every year of age, and white British ethnicity $(p<0.01)$.

Conclusion Older patients and those of white British origin are particularly at risk for developing $\mathrm{HE}$ and should be monitored carefully following the TIPS procedure. The finding that patients with hypernatraemia have significantly reduced survival rates is novel; it most likely reflects overdiuresis and should be corrected pre-procedure. Thus, careful assessment and selection of candidates for TIPS insertion for refractory ascites as well as closer and longer-term monitoring may help prevent the development of $\mathrm{HE}$ and lead to improved outcomes.

Disclosure of Interest None Declared.

\section{PWE-124 VAP-1 ACTIVITY IS ELEVATED IN PSC AND MODULATES A4B7-DEPENDENT LYMPHOCYTE ADHESION TO HSEC UNDER FLOW}

doi:10.1136/gutjnl-2013-304907.412

1."P J Trivedi, ' $\mathrm{C}$ Weston, ${ }^{1} \mathrm{C}$ Corbett, 'E Liaskou, 'D Adams. 'Centre for Liver Research and NIHR Biomedical Research Unit, University of Birmingham, Birmingham, UK

Introduction Vascular adhesion protein (VAP)- 1 is an adhesion molecule which possesses potent amine oxidase activity, and deaminates dietary amines resulting in the production of $\mathrm{H}_{2} \mathrm{O}_{2}$. Through this function, VAP-1 leads to activation of $N F \kappa B$ in hepatic sinusoidal endothelium (HSEC) resulting in the expression of mucosal-vascular cell-adhesion molecule-1 (MAdCAM-1); a mechanism proposed to contribute to the homing of gut-tropic lymphocytes expressing $\mathrm{a} 4 \mathrm{~b} 7$ to the liver. Given the putative role this pathway has in hepatic diseases complicating inflammatory bowel disease (IBD), we set out to quantify circulating/soluble (sVAP-1) and intrahepatic VAP-1 enzyme activity in primary sclerosing cholangitis (PSC), and evaluate the functional consequence of its inhibition on MAdCAM-1 dependent lymphocyte recruitment to HSEC.

Methods Total VAP-1 concentration was measured by ELISA VAP-1 amine oxidase activity was quantified in human serum and explanted liver tissue using the amplex red assay. Flow-based adhesion assays were performed using human HSEC isolated from liver explants, activated with TNFa and methylamine (VAP-1 substrate), and treated with VAP-1 antibody or semicarbazide (VAP-1 enzyme inhibitor). FAC-sorted peripheral blood leucocytes expressing a $4 \mathrm{~b} 7$ were perfused over HSEC under flow rates simulating physiological shear $(0.05 \mathrm{kPa})$ and adhesion and transmigration quantified.

Results Patients with PSC had significantly higher circulating median VAP-1 enzyme activity (114.5 $\mathrm{pmol} \mathrm{H}_{2} \mathrm{O}_{2}$ produced/min/ $\mathrm{ml}$ serum, IOR 100.6-134.7) than patients with IBD (60.3, IOR 38.5-73.0; $P=0.006$ ), normal controls (84.0, IOR 77.7-105.7; $P=0.020)$ and individuals with $\mathrm{PBC}$ (53.9, IOR 33.0-90.9; $P=0.006)$, and trended higher than AIH (77.6, IOR 51.0-124.5; $P=0.200)$ (Mann-Whitney). Total sVAP-1 concentration correlated well with sVAP-1 enzyme activity $\left(R^{2}=0.75\right)$. Intrahepatic median VAP-1 activity was also significantly higher in PSC $(97.6 \mathrm{pmol}$ $\mathrm{H}_{2} \mathrm{O}_{2} / \mathrm{min} / \mathrm{mg}$ protein respectively, IOR 69.5-114.5) vs. PBC (24.6, IOR 18.7-27.8; $P=0.029)$ and AIH (32.3, IOR 23.3-35.6; $P=0.028)$ (Mann-Whitney). HSEC pretreatment with semicarbazide but not antibody led to a profound reduction in total a $4 \mathrm{~b} 7^{+}$lymphocyte adhesion (75\%); however, both antibody and enzyme inhibition independently reduced transmigration by $\sim 50 \%$ compared to untreated HSEC.

Conclusion sVAP-1 enzyme activity is greater in PSC compared to IBD alone, normal controls, and other immune-mediated liver diseases. Intrahepatic VAP-1 enzyme activity is significantly higher in PSC compared to AIH and PBC. Inhibition of VAP-1 leads to abrogation of a4b7-mediated adhesion to HSEC, representing a putative target for therapeutic intervention in PSC.

Disclosure of Interest None Declared.

\section{PWE-125 STRATIFICATION OF HEPATOCELLULAR CARCINOMA RISK IN PRIMARY BILIARY CIRRHOSIS BY BIOCHEMICAL RESPONSE TO TREATMENT}

doi:10.1136/gutjnl-2013-304907.413

1."P Trivedi, ${ }^{\prime} \mathrm{K}-\mathrm{K} \mathrm{Li},{ }^{1} \mathrm{~T}$ Bruns, ${ }^{1} \mathrm{H}$ Shah, ${ }^{2} \mathrm{D}$ Tripathi, ${ }^{2 \mathrm{~T}}$ Shah, ${ }^{2} \mathrm{~J}$ Neuberger, ${ }^{1} \mathrm{G}$ Hirschfield. ${ }^{1}$ Centre for Liver Research and NIHR Biomedical Research Unit, University of Birmingham; 'Liver Unit, Queen Elizabeth Hospital, University Hospitals Birmingham, Birmingham, UK

Introduction Aims: To review patterns of hepatocellular carcinoma (HCC) presentation in patients with primary biliary cirrhosis $(\mathrm{PBC})$ in the context of disease severity and biochemical response to treatment.

Methods Patients with confirmed PBC seen at the Queen Elizabeth hospital (Birmingham) between September 1996 and December 2012 were identified, and a systematic retrospective chart review performed. Potential risk factors for the development of HCC were analysed using Fisher's exact test, multivariate logistic regression and Kaplan-Meier estimates (GraphPadv5.03).

Results Of 397 patients with PBC identified, 221 patients had developed cirrhosis, and 30 presented with or developed HCC (median age 68) over a 16 year period. Of all cases of HCC, 21 (70\%) were identified through HCC surveillance programmes, and 19 patients presented within Milan criteria. In our practise, HCC was exclusively seen in the presence of cirrhosis. Using the cirrhotic nonHCC group as our comparator, male cirrhotics were more likely to develop HCC than female cirrhotics (OR 3.32; 95\% CI 1.34-8.26; $P=0.012$ ), and those with HCC were older at the time of diagnosis of cirrhosis (68 years vs. 57 years; $P=0.001) .67 \%(n=20)$ in the HCC group and $76 \%(n=145)$ of the non-HCC cirrhotic group were taking UDCA for $>1$ year following diagnosis of $\mathrm{PBC}(P=0.21)$. Of the patients taking UDCA for $>1$ year, $52 \%(n=76)$ of the nonHCC cirrhotic group were biochemical responders according to the original Corpechot criteria compared with $20 \%(n=4)$ of the HCC group $(P<0.0001)$. Not taking or non-response to UDCA was significantly associated with the development of HCC (OR 4.54; 95\% CI 2.24-9.20; $P<0.0001$ ), and remained a significant risk factor after restricting the analysis to UDCA non-responders (OR 4.33; 95\% CI 2.31-8.12; $P<0.0001)$. The cumulative hazard for HCC in cirrhotic UDCA non-responders was 0.18 at 5 years and 0.35 at 10 years after diagnosis of cirrhosis, but $<0.1$ after 10 years in UDCA responders $(P=0.003)$. The overall HCC incidence rate for cirrhotic patients with $\mathrm{PBC}$ was 3.4 cases $/ 100$ patient-years. When stratified by treatment response, UDCA responders had HCC rates of only 1 case/100 patient years, compared to 5 cases $/ 100$ patient-years in nonresponders.

Conclusion Development of HCC in PBC is associated with a failure to respond to therapy with UDCA, older age at diagnosis of cirrhosis and male gender. The incidence rate for HCC development in cirrhotic patients responding to UDCA is arguably beneath the point at which cost-efficacy is likely met.

Disclosure of Interest P. Trivedi Grant/Research Support from: Wellcome Trust funded clinical research fellow, K.-K. Li: None Declared, T. Bruns: None Declared, H. Shah: None Declared, D. Tripathi: None Declared, T. Shah: None Declared, J. Neuberger: None Declared, G. Hirschfield: None Declared 